\title{
Up-down asymmetry of type I plasma waves in the equatorial electrojet region
}

\author{
S. Ravindran, B. V. K. Murthy \\ Space Physics Laboratory, Vikram Sarabhai Space Centre, Trivandrum 695 022, India \\ Received: 5 June 1996 / Revised: 6 January 1997 / Accepted: 13 January 1997
}

\begin{abstract}
The up-down asymmetry of the type I plasma waves in the equatorial electrojet region during daytime is investigated. It is shown that the asymmetry is exhibited not only in the vertical direction but also is manifested in the oblique direction $\left(24.6^{\circ}\right.$ off zenith). The results further indicate the effect of neutral winds on the phase velocities of type I plasma waves.
\end{abstract}

\section{Introduction}

Using the VHF backscatter radar with nearly vertically ( $\sim 1^{\circ}$ off zenith to satisfy aspect sensitivity) pointing antenna beam at Jicamarca, it has been reported that the radar signal returns corresponding to upward propagating type I waves are stronger and more common than the downward propagating type I waves in the daytime when the ambient primary electric field $\left(E_{y}\right)$ is eastward and vice versa in the nighttime when $E_{y}$ is westward (e.g. Fejer et al., 1976; Farley et al., 1978; Kudeki et al., 1985). Kudeki et al. (1985) explained the observed updown asymmetry of type I waves as due to currents associated with large-scale primary waves and second harmonic distortion of these waves. Balsley (1970) has reported east-west asymmetry in the electron drift velocity observed at Jicamarca. Farley (1985) has suggested this asymmetry to be a geographical rather than a plasma effect.

Using the VHF radar at Trivandrum $\left(8^{\circ} 33^{\prime} \mathrm{N}, 77^{\circ} \mathrm{E}\right.$, dip $0.5^{\circ} \mathrm{N}$ ) with antenna beams which can be switched from $24.6^{\circ}$ off zenith westwards, to zenith and to $24.6^{\circ}$ off zenith eastwards, we have studied east-west asymmetry of type I plasma waves in addition to their updown asymmetry. The east-west asymmetry in the strength of type I waves is interpreted as a manifestation of the up-down asymmetry. The results of this study are presented here.

Correspondence to: B. V. Krishna Murthy

\section{VHF radar system at Trivandrum}

The basic system details of the VHF radar operating at 54.95 MHz were presented in Reddy et al. (1987) and are not repeated here. In the present study, in place of the regular $16 \times 4$ Yagi antenna array with a fixed beam orientation of $30^{\circ}$ off zenith westwards, we have used a $15 \times 4$ antenna array which has provision to switch the antenna beam in the east-west plane to three directions, namely $24.6^{\circ}$ off zenith westwards and eastwards and zenith. The $15 \times 4$ Yagi antenna array uses interlaced phasing to achieve beam switching. The details of this antenna array are presented in Reddy et al. (1990). The beam width (2-way) of the oblique beams is $\sim 3.3^{\circ}$ and that of the vertical beam is $\sim 3^{\circ}$.

\section{Results and discussion}

The VHF radar observations were carried out during daytime on four days, October 12, 1988, September 20, 1990, October 9, 1990 and January 5, 1993 using the three antenna beam orientations. All the four days are magnetically quiet days with an $A_{p}$ index $<17$. In the present study only Doppler spectra corresponding to type I plasma waves are considered. The presence of type I in the Doppler spectra is identified by the welldefined narrow peak at a frequency corresponding to a velocity close to ion acoustic velocity. The zeroth and first moments of the Doppler spectra (type I) are calculated which give the power of the signal return and mean Doppler frequency $f_{1}$ of the type I irregularities respectively.

Typical Doppler spectra observed with vertical and oblique antenna beams are shown in Figs. 1 and 2 respectively. In these figures, the peak corresponding to the type I Doppler frequency is indicated by an arrow.

In Fig. 1 both up-going (negative Doppler) and down-going (positive Doppler) type I waves are seen to be present except at 1010 hours when only the upgoing wave is seen. When both are present (in the same Doppler spectrum) the peak power of the up-going 


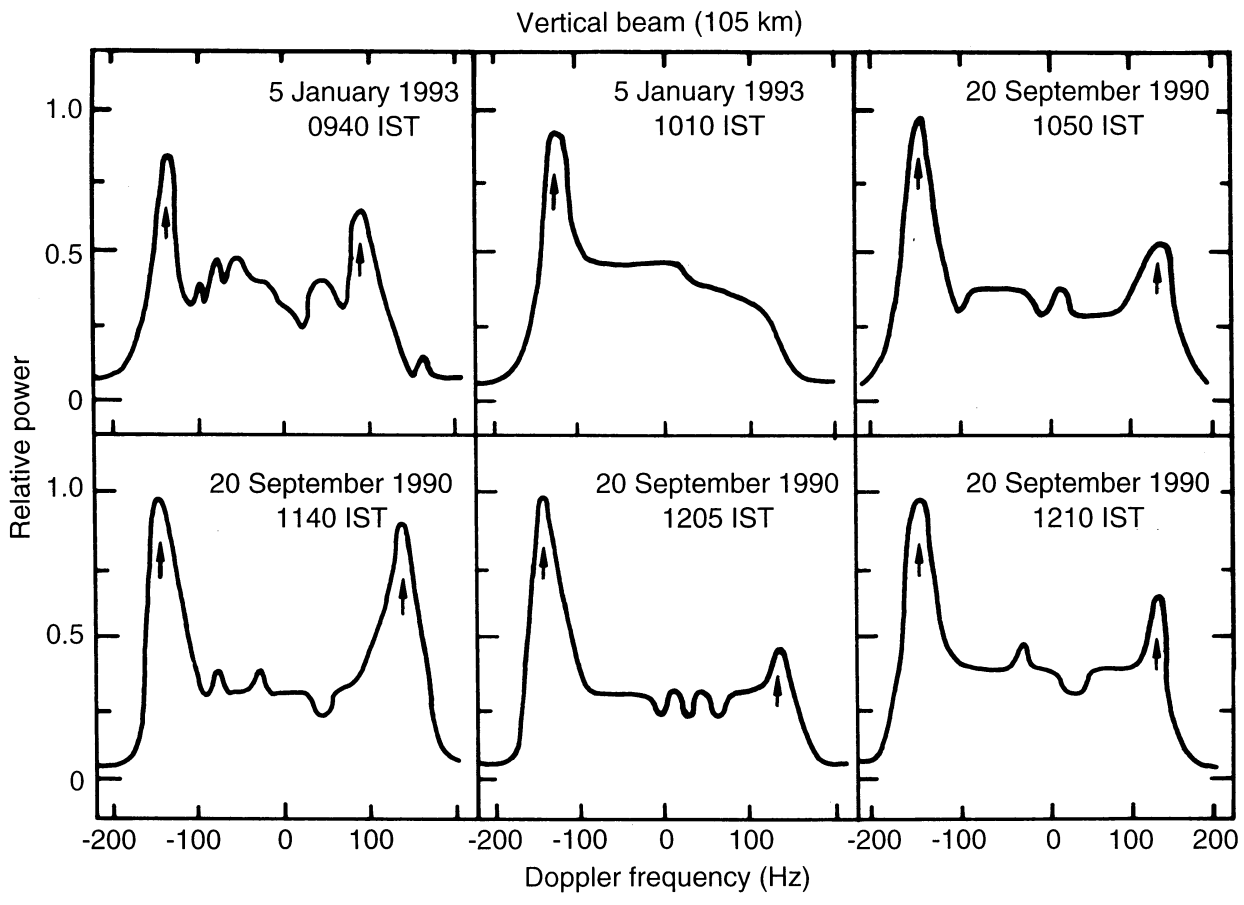

Fig. 1. Sample Doppler spectra for vertical antenna beam waves is greater than that of the down-going waves. During daytime when the electron flow (and hence irregularity drift) is westward (corresponding to eastward $E_{y}$ ), the west-oriented beam will be seeing plasma waves with upward inclined propagation vectors along the line-of-sight and the east oriented beam those with downward inclined propagation vectors (Cohen and Bowles, 1967). From Fig. 2, it is clear that type I signatures appeared in the spectra corresponding to both the beams. However, the spectral peak corresponding to the west beam is generally stronger than that corresponding to the east beam.

The peak power and mean Doppler frequency of type I for the vertical beam at different times at three altitudes on September 20, 1990 are shown in Fig. 3a,b. It is clear that at the higher and lower altitudes of 108 and $102 \mathrm{~km}$ the up-going waves occur more frequently than the down-going ones whereas at $105 \mathrm{~km}$ the occurrences of the two are equal (Fig. 3a). Over the three altitudes, the up-going waves are (often) stronger than the down-going ones as indicated by the power of the signal return. No significant differences are seen, in general, between the mean Doppler frequency of the up and down-going waves (Fig. 3b). Similar features are revealed on the other three days of observations (not shown here).

The peak power and mean Doppler frequency corresponding to the west and east beams at different times on January 5, 1993 at three altitudes are shown in Fig. 4a,b respectively. The number of occurrences of type I waves is, in general, greater for the west beam than for the east beam (Fig. 4a). Considering the mean Doppler frequency $\left(f_{1}\right)$, the variations are similar for the west and east beams and the values (of $f_{1}$ ) are nearly the same. At $104 \mathrm{~km}$, while the variations of $f_{1}$ are similar, $f_{1}$ values are greater for the west beam than for the east beam at times later than $1000 \mathrm{~h}$. The differences between the two reach values as high as $80 \mathrm{~m} / \mathrm{s}$ (velocity scale is shown along the ordinate on the right hand side). At $107 \mathrm{~km}$ the behaviour of $f_{1}$ is somewhat similar to that at $104 \mathrm{~km}$. Similar features are revealed on other three days of observations (not shown here). Considering all the four days of observations, it is seen that $f_{1}$ is different for west and east beams on a number of occasions.

The results for the four days of observations are summarized in Table 1, considering the data at the three altitudes. The number of occurrences (of type I) for the west beam (waves with upward inclined propagation vectors) are about $25 \%$ more than that for the east beam (waves with downward inclined propagation vectors). For the data of the vertical beam, the number of occurrences of up-going waves is about $77 \%$ greater than that of down-going waves. The number of cases in which the peak power $P_{I}$ for up-going waves is greater than that for the down-going waves is more than the number of cases in which $P_{I}$ for down-going is greater than that for up-going waves. In about $30 \%$ of the cases the two powers are same.

The results presented clearly bring out the preponderance of the occurrence of up-going type I plasma waves over the down-going waves in the daytime as well as that of upward inclined waves over the downward inclined waves. This difference is more marked for the vertical beam (upward and downward going waves) than for the west and east beams (waves with upward inclined and downward inclined propagation vectors). Further, the upward going (and upward inclined) waves are, in general, stronger than the downward going (and downward inclined) type I plasma waves.

Kudeki et al. (1985) showed from theoretical considerations that the up-down asymmetry of type I plasma waves is a consequence of the non-linear development of 


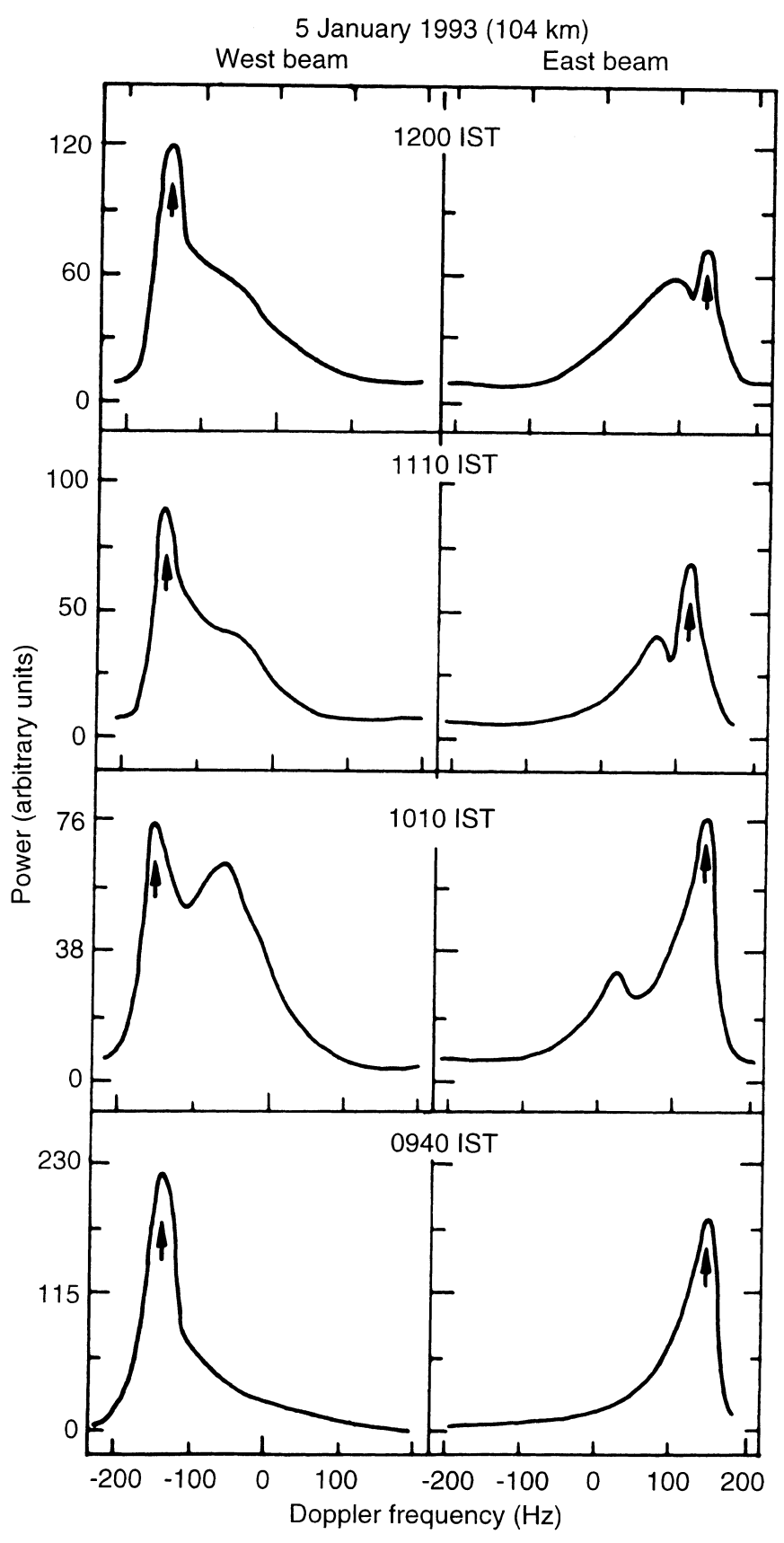

Fig. 2. Sample Doppler spectra for west and east antenna beam

the horizontally propagating large-scale primary waves. In the daytime equatorial electrojet, the vertical electron motion associated with the large-scale horizontally propagating primary wave causes a net downward motion of the electrons because the downward motion of the regions of enhanced electron density involves more electrons than the upward motion of the regions of depleted electron density. This net vertical motion would disappear in the presence of a balancing asymmetry in the vertical velocity waveform of the primary wave resulting in a reduction of the vertical polarization field. Such an asymmetry would lead to the preponderance of the up-going type I waves over the down-going waves. Further, Kudeki et al. (1985) have shown that the non-linear deformation of the perturbed vertical velocity of electrons (second harmonic distortion) accentuates this asymmetry.

The observations of up-down asymmetry of type I waves in the daytime presented are in accordance with the explanation of Kudeki et al. (1985). We have also shown that this asymmetry exists even when looking in an oblique direction $\left(24.6^{\circ}\right.$ off zenith) with upward inclined waves being predominant over the downward inclined waves which is also in qualitative agreement with the explanation of Kudeki et al. (1985). However, the effect of reduction of vertical polarization field (due to large-scale primary waves as mentioned already) would be more on the vertical velocity of the electron velocity (relative to ions) than on the oblique components. Thus the up-down asymmetry can be expected to be more dominant in the vertical beam observations than in the oblique beam observations as observed in the present study.

It is seen in the present study that $f_{1}$ is significantly more for the west-beam than for the east-beam on some occasions (e.g. on January 5, 1993, Fig. 4b). It is known that neutral winds affect the phase velocity of the type I waves (e.g. Fejer et al., 1975; Cohen and Hooke, 1978). Neutral atmospheric motions including prevailing winds, atmospheric tides and atmospheric gravity waves can be expected to produce changes in the phase speed of the type I plasma waves (Cohen and Hookes, 1978). The observed phase speed $\left(V_{p}\right)$ of the type I waves can be written as (Balsley et al., 1976)

$V_{p}=C_{s}+V_{y} \cos \theta$

where $C_{s}$ is the ion-acoustic speed, $\theta$ is the elevation and $V_{y}$ is the zonal speed of neutral wind. Thus, the difference in the observed type I phase speeds in the east and west directions can be attributed to east-west asymmetry in the neutral motions. That is, the zonal wind at the observation volume seen by the radar for the east and west beams could be different. Balsley (1977) has also reported strong longitudinal non-uniformity in the neutral wind structure over Jicamarca. It may be noted that the horizontal separation of the observation volume of the east and west beams at $100 \mathrm{~km}$ altitude for the radar configuration in the present study is $\sim 90$ $\mathrm{km}$. It is interesting note that the peak power $P$ (for the same beam) has not shown in general significant differences (between west and east beam observations) in contrast to $f_{\mathrm{I}}$. As neutral winds do not affect the strength of the type I waves (Fejer et al., 1975), attributing the observed east-west differences in $f_{\mathrm{I}}$ to neutral (zonal) wind differences is consistent with this.

The phase velocity $V_{p}$ data on January 5, 1993 (Fig. 4b) in the prenoon period (when the data is continuous) is subjected to fast Fourier transform (FFT) to delineate periodic components. It is found that $V_{p}$ corresponding to both the beams reveals periodic components with periods of $\sim 60$ and $\sim 30 \mathrm{~min}$ at all three altitudes with amplitudes $\sim 10 \mathrm{~m} / \mathrm{s}$ and $5 \mathrm{~m} / \mathrm{s}$ respectively and no significant phase difference between corresponding periodic components from west and east beam data. The difference in the steady component is 

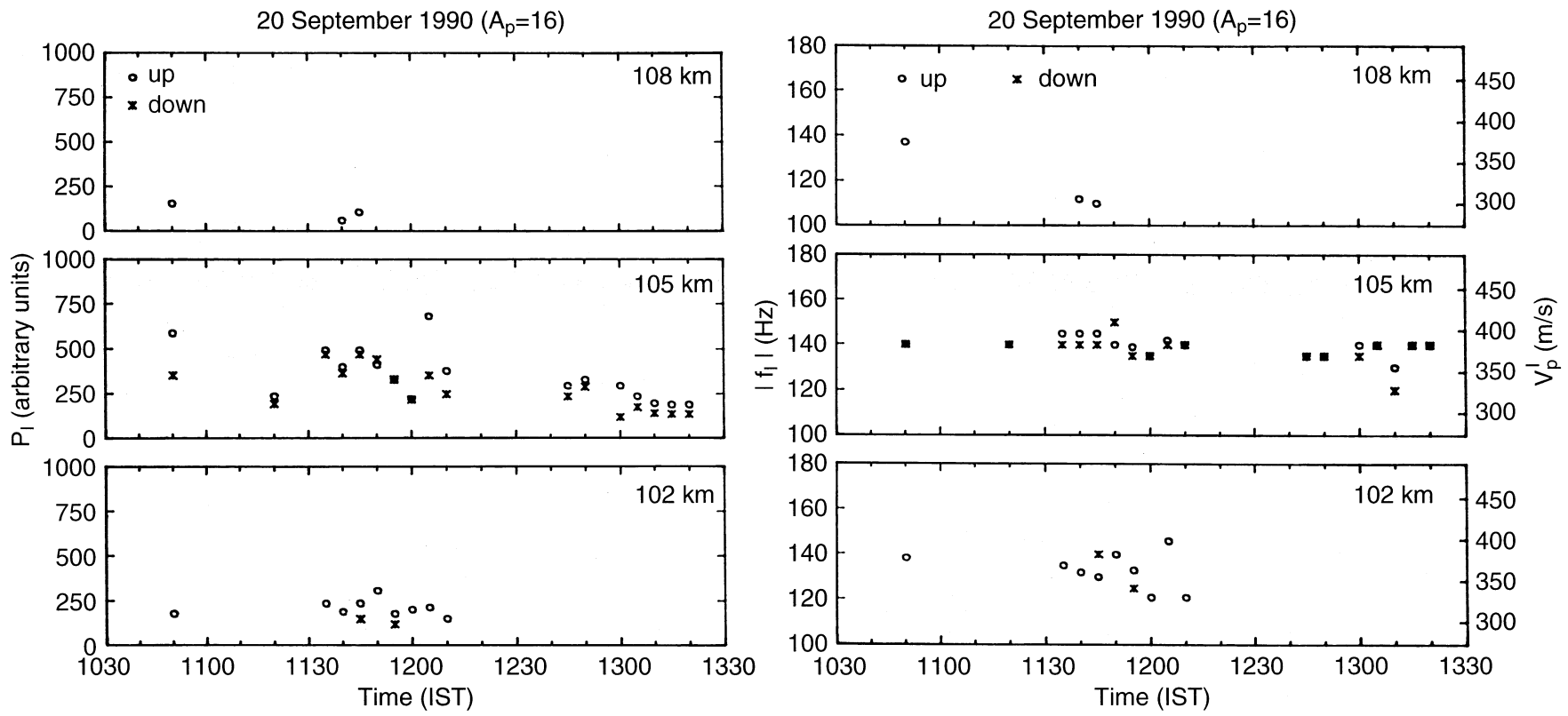

Fig. 3. Backscatter signal power and peak Doppler frequency for up-going and down-going type I plasma waves
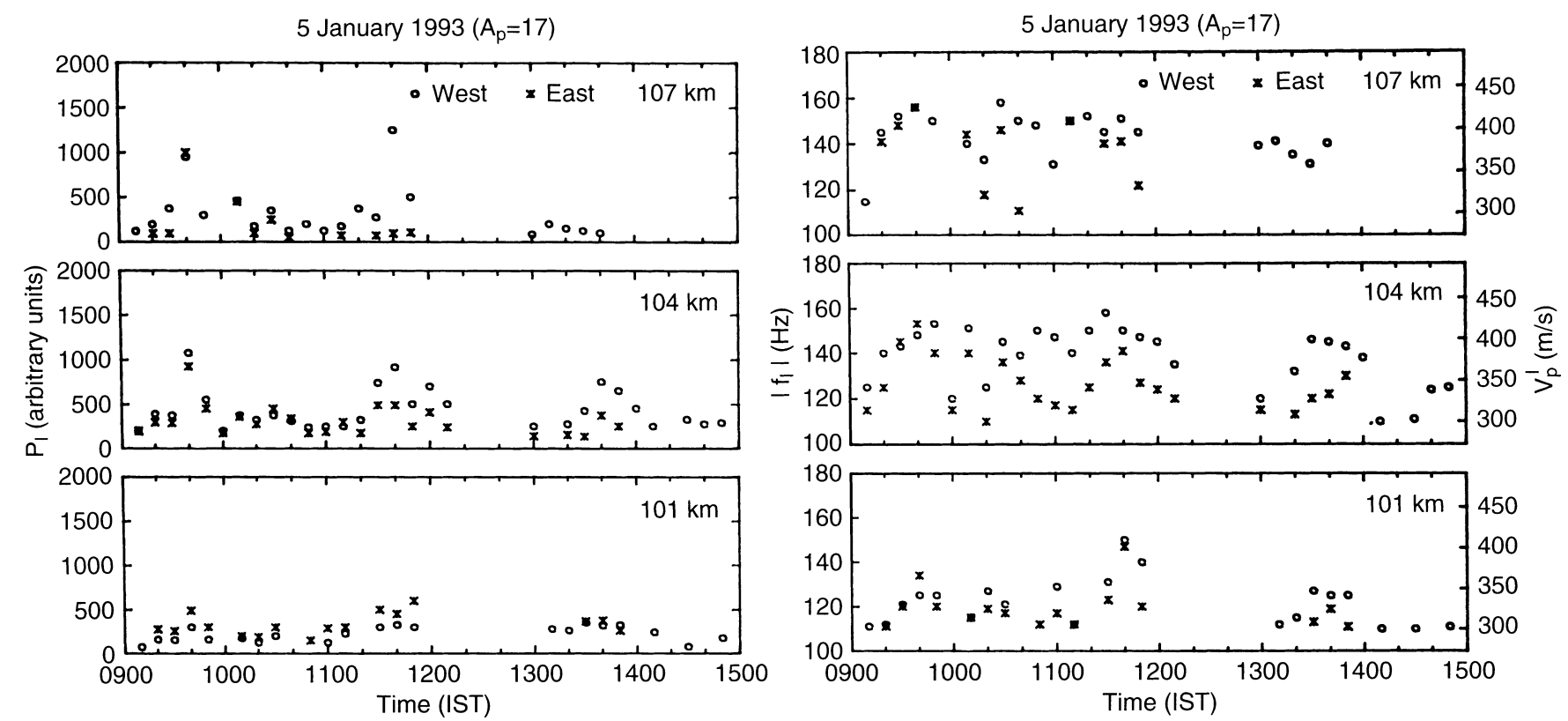

Fig. 4. Backscatter signal power and peak Doppler frequency for type I waves with upward inclined (west antenna beam) and downward inclined (east antenna beam) propagation vectors

Table 1.

\begin{tabular}{lllllll}
\hline \multirow{2}{*}{$\begin{array}{l}\text { Antenna } \\
\text { position }\end{array}$} & \multicolumn{2}{l}{ Number of occurrences } & & \multicolumn{4}{l}{ Number of cases for which } \\
\cline { 2 - 3 } & Upgoing & Downgoing & & $\mathrm{P}_{1}(\mathrm{U})>\mathrm{P}_{1}(\mathrm{D})$ & $\mathrm{P}_{1}(\mathrm{U})<\mathrm{P}_{1}(\mathrm{D})$ & $\mathrm{P}_{1}(\mathrm{U})=\mathrm{P}_{1}(\mathrm{D})$ \\
\hline $\mathrm{E}-\mathrm{W}$ & 164 & 131 & 54 & 31 & 38 \\
$\mathrm{~V}$ & 62 & 35 & 21 & 3 & 10 \\
\hline
\end{tabular}

$\mathrm{P}_{1}(\mathrm{U})$, power in upgoing type $\mathrm{I}$ waves

$\mathrm{P}_{1}(\mathrm{D})$, power in downgoing type I waves 
$\sim 10 \mathrm{~m} / \mathrm{s}$ which could be due to a longer period component (longer than the data length). These values appear to be reasonable to be attributed to the effect of neutral winds.

Acknowledgements. We wish to express our thanks to Dr CA Reddy for his involvement in the initial stages of this work. We thank Mr KV Janardhanan and his group in the Space Physics Laboratory for carrying out the VHF backscatter radar experiment. We thank Mr PG Jayaprakash for word processing of this paper.

Topical Editor D. Alcaydé Thanks M. A. Abdu and E. Kudeki for their help in evaluating this paper.

\section{References}

Balsley, B. B., A longitudinal variation of electron drift velocity in the equatorial electrojet, J. Geophys. Res., 75, 4291, 1970

Balsley, B.B., E-region dynamics, J. Atmos. Terr. Phys., 39, $1087-$ 1096, 1977

Balsley, B. B., B. G. Fejer, and D. T. Farley, Radar measurements of neutral winds and temperatures in the equatorial $\mathrm{E}$ region, J. Geophys. Res., 81, 1457-1459, 1976

Cohen, R., and W. H. Hooke, Neutral atmospheric motions manifested in radar echo Doppler shifts from two-stream irregularities in the equatorial electrojet, J. Geophys. Res., 83, 4791-4797, 1978
Cohen, R., and K. L. Bowles, Secondary irregularities in the equatorial electrojet, J. Geophys. Res., 72, 885-894, 1967

Farley, D. T., Theory of equatorial electrojet plasma waves: new developments and current status, J. Atmos. Terr. Phys., 47, 729744, 1985

Farley, D. T., B. G. Fejer, and B. B. Balsley, Radar observations of two dimensional turbulence in the equatorial electrojet, 3 . Night time observations of type I waves, J. Geophys. Res., 83, 56255632, 1978

Fejer, B. G., D.T. Farley, B .B. Balsley and R. F. Woodman, Oblique VHF radar spectral studies of the equatorial electrojet, J. Geophys. Res., 80, 1307-1312, 1975

Fejer, B. G., D. T. Farley, B. B. Balsley, and R .F. Woodman, Radar observations of two-dimensional turbulence in the equatorial electrojet, 2, J. Geophys. Res, 81, 130-134, 1976

Kudeki, E., D. T. Farley, and B. G. Fejer, Theory off spectral asymmetries and nonlinear currents in the equatorial electrojet, J. Geophys. Res., 90, 429-436, 1985

Reddy, C. A., B. T. Vikram Kumar, and K. S. Viswanathan, Electric fields and current in the equatorial electrojet deduced from VHF radar observations, 1. A method of estimating electric fields, J. Atmos. Terr. Phys., 49, 183-191, 1987

Reddy, C. A., K. V.Janardhanan, K. K. Mukundan and K. S. V. Shenoy, Concept of an interlaced phased array for beam switching, IEEE Trans. Antennas and Propag., 38, 573-575, 1990 